\title{
Management Information System for Compensation under Multihoming Network Architecture
}

\author{
Cao Yuan (iD ${ }^{1}$ and Hua Yicun (iD $^{2}$ \\ ${ }^{1}$ Human Resource Department, Donghua University, Shanghai 201620, China \\ ${ }^{2}$ The College of Information Science and Technology, Donghua University, Shanghai 201620, China
}

Correspondence should be addressed to Hua Yicun; huayicun@dhu.edu.cn

Received 15 April 2021; Revised 27 October 2021; Accepted 5 November 2021; Published 20 November 2021

Academic Editor: Nawab Muhammad Faseeh Qureshi

Copyright (C) 2021 Cao Yuan and Hua Yicun. This is an open access article distributed under the Creative Commons Attribution License, which permits unrestricted use, distribution, and reproduction in any medium, provided the original work is properly cited.

\begin{abstract}
The management information system for compensation under multihoming network architecture has been developed in order to improve the time efficiency, accuracy, and level of informatization of compensation management in university and deal with the rising data and difficulty in exchanging information between various management information systems resulting from the changing compensation policies. This system is designed based on multihost data network architecture, including function modules of all kinds of compensation promotion, personnel historical data management, time warning, statistics, and report generation. This system integrates my five years' experience in the front-line of compensation management work. The purpose is to fully solve the practical problems of compensation management in universities, truly help the work of compensation administrators, unify the fragmented compensation management works, and comprehensively improve the level of compensation management. It has a strong popularization and signification for reference for the compensation management work of similar universities.
\end{abstract}

\section{Introduction}

Compensation information management and statistical analysis is an important routine work of personnel department; the quality of data management directly affects the efficiency and quality of personnel management work [1]. Compensation information has the characteristics of fast update, complex, and trivial. The practical management working process is often faced with serious data redundancy, not timely update, repetitive work, sharing difficulties, and other problems. Information cannot be scientific management, and maintenance [2] will greatly reduce the efficiency and quality of personnel management.

Public institution is a kind of social service organization for the purpose of public welfare in China, which is engaged in education, science and technology, culture, health, and other activities. Most colleges and universities [3] are public institutions, and the compensation management of public institutions has a strong policy focus. In short, the compensation of public institutions is composed of national com- pensation, uniformly prescribed allowance, local allowance, and institution allowance. The first three parts are uniformly prescribed by national or provincial policies. All public institutions within the scope of policy are subject to uniform references. All national public institutions have the same reference of national compensation, and all public institutions in the same province have the same reference of some provincial allowance. The institution allowance is the only part determined by the institution itself. Therefore, we can find that the compensation of public institutions has a strong continuity, especially the national compensation, which has the same reference all over the country.

For university faculty, all their experiences since they went to university will affect the national compensation and local allowance. Therefore, the compensation management in university is complex. It is necessary to have all the study and work records of each staff to determine their compensation. With the growing number of talents in universities and the rapid development of internet and information technology, compensation management has entered the 
digital era, and the informatization construction of universities has been further promoted. As the essential tool of compensation management, a management information system (MIS) for compensation plays a positive role in the management of universities. At the same time, the compensation management in university is characterized by strong policy, large amount of data, and detailed report forms and request highly accurate and safe. Thus, a flexible and efficient MIS for compensation which can make the compensation management work tends to be more scientific, standardized, and modern urgently needs to be developed.

The traditional host network or end users usually only connect to the content source network through a single path. When the only exit path fails, the whole client network cannot get the content source. This problem can be solved by using multihoming technology [4]. The client network can be connected to the outside world through multiple outlets, such as WiFi network [5] and 4G network [6]. When one network fails, it can also transmit data through other networks. In the real network transmission, especially in the big data network [7], the multihoming technology can not only ensure the transmission stability but also improve the transmission efficiency. The client network can simultaneously transmit data through multiple network paths to increase the transmission speed or select the shortest exit of the transmission destination path to reduce the transmission time.

The multihoming technology under TCP/IP architecture [8] has been relatively mature. The classic solutions include host multihoming technology, transport layer multihoming technology, and network layer multihoming technology. Host multihoming technology mainly uses the large address space in IPv6 [9] to communicate with different ISPs depending on multiple IP addresses owned by each host, so as to obtain content from multiple paths. For example, in multiple care of address registration (MCoA [10]), the mobile host or user can register multiple care of addresses for the home address, create multiple binding cache entries, number them with a new binding ID, and send this message in the binding update. Transport layer multihoming technology mainly refers to the design of a new transport protocol supporting multihoming technology to replace TCP technology. For example, Dreibholz et al. designed a new transport layer protocol: flow control transport protocol (SCTP [11]). SCTP is a unicast protocol that supports data exchange between two endpoints and allows each endpoint to have multiple IP addresses. Network layer multihoming technology mainly refers to the technology of inserting a new protocol stack between network layer and transport layer. The representative of network layer multihoming technology is host identity protocol (HIP [12]) and Shim6 [13]. These technologies complete the mapping from a single host name to multiple network addresses by introducing a new namespace, and transition between network layer and transport layer, so as to achieve multihoming transmission.

Based on the practical work of compensation management in our university, this paper takes the digital campus construction [14] as an opportunity to develop a management information system for compensation under multihoming network architecture (MNC-MIS). Based on the in-depth study of the characteristics of compensation management in university and the composition of personnel information, through the analysis and research on the types, structures, and characteristics of our university faculty information, MIS for compensation management is designed, which adapt to the characteristics of university and provided an information platform for university compensation management, analysis, and decision-making. The main contributions are as follows:

(1) The multihoming network architecture of MIS for compensation is designed: this MIS is based on the construction of digital campus. This paper presents the multihoming network architecture of MIS for compensation. Users can obtain compensation management information through LAN, IPv4, IPv6, and 4G

(2) This paper designs a MIS for compensation to adapt to the characteristics of university compensation management: the overall structure of the system is divided into four layers: interaction layer, application layer, data layer, and external layer. In the external layer, the MIS systems that need compensation data support can interact with each other through the unified data bus format. The interaction layer mainly includes the program modules that need to interact with users, which is the entrance and exit of the system. The application layer processes all data management, and the data layer stores system data

(3) The function module design of MNC-MIS is given: including all kinds of function modules of compensation promotion, all kinds of function modules of time warning, function module of personnel history data management, and all kinds of function modules of statistics and report generation. The functions and details of each module are explained

(4) The data flow of MNC-MIS is designed: taking the practical work of new teachers in colleges and universities, the determination and adjustment of social security payment base, the process of monthly compensation issuing and summarizing, and the whole life cycle management as examples, this paper explains the flow and operation of the system data

In the remainder of this paper, problems of the existing compensation data management system are discussed in Section 2. The design of the management information system for compensation under multihoming network architecture is given in Section 3. Section 4 presents the function and effect of MNC-MIS. Finally, the conclusions and future research are provided in Section 5.

\section{Problems of the Existing Compensation Data Management System}

At present, there are not many colleges and universities that have developed MIS for compensation. There are six major problems of existing compensation management systems. 
(1) Data storage and management methods are very backward

The compensation data management of many colleges and universities is mainly composed of paper files and electronic files. These electronic files are also basically electronic documents formed by office software such as word or excel. They are operated on a single machine and can achieve information sharing through U-disk or e-mail, which causes problems such as inaccurate data, repeated processing of information, and difficult to achieve real-time sharing. The existing compensation data management systems in colleges and universities are different, and there is no effective management mode. The commercial development of MIS for compensation is mainly for enterprises and [15], which is expensive, and has full functions but lacks personalized demand, which is difficult to meet the needs of compensation data management in universities.

\section{(2) Salary data cannot be exchanged}

The existing compensation management system is selfclosed. In the existing system, the common problem is that all kinds of systems are independent of each other and lack of information automatic conversion and sharing function. Operators need to switch back and forth between various systems frequently, which is the inevitable result of the single machine era. Although a large amount of data has been accumulated and collected formally, the repeated storage of these original data has not been scientifically sorted and classified, which brings not only information but also information garbage. Therefore, it is difficult to extract valuable composite information from it.

(3) The workload of information maintenance is large

The information age is full of competition. System developers usually adopt short, flat, and fast system design schemes, lacking overall consideration and optimal combination, resulting in repeated investment of many resources and waste of financial resources, increase the amount of redundant information, occupy storage space, and increase the amount of maintenance. On the other hand, the amount of maintenance comes from the C/S structure of the system itself [16], which is characterized by maintaining both the server and the client.

(4) Lack of big data analysis and auxiliary salary decision-making functions

Management and decision-making are inseparable, but people often ignore the important link of decision-making when developing the system. Compensation management software only provides the daily business, ignoring such important information as the comparison between the growth rate of annual total income and the average compensation level of the society in the same period, the analysis of the salary distribution ratio of all kinds of personnel, the relationship between labour costs and benefits, etc. These data are usually the key to assist decision-making, but they cannot be obtained directly. This is a serious system functional defect.
(5) Lack of automatic reminder function

Current systems usually do not prompt managers what work they should pay attention to, such as a teacher who is close to retirement age and needs to calculate the retirement compensation. Or when the time for unified compensation adjustment is up, you need to modify compensation data in batch. The resulting mistakes often affect the working mood of both sides.

(6) The visualization of management information is poor

At present, the informatization reform of university is still in the exploratory stage, and the degree of management information visualization is low. In the process of information management in universities, problems such as lack of data, noise, and unstructured are often faced, which hinder the information decision-making.

Nowadays, there are many kinds of MIS for compensation management in the market, but most of them are not practical enough, especially the MIS for compensation management in universities. There are many defects such as incomplete function, poor report processing function, complicated query, and statistics. At the same time, the current compensation management system used by our school was developed in the 1990s, which requires low versions of computer hardware and software. Its development background is based on the postcompensation system of the 1993 compensation policy. Obviously, the system cannot adapt to the rapid development of computer science and technology and also cannot meet the requirements of a merit pay system. Therefore, a new MIS for compensation has to be developed.

\section{Design of Management Information System for Compensation under Multihoming Network Architecture}

3.1. Multihoming Network Architecture. There is a large amount of compensation management data in universities, and the real-time requirement of data processing is high. In this paper, combined with the actual compensation management work of our school, taking the digital campus construction as an opportunity, we develop the MNC-MIS. The schematic diagram of the multihoming data network structure is shown in Figure 1.

The network architecture is divided into three layers: data source, multihoming network, and terminal processor. Among them, the data source stores compensation management data, the multihoming network includes $4 \mathrm{G}$ or $5 \mathrm{G}$ network [17], LAN [18], IPv4, and IPv6 [19], and the terminal processor can be PC, smartphone, or tablet computer [20]. The advantage of multihoming network architecture is that when one of the network links is disconnected, the end users can still get the data source through other host networks, which will not affect the development of compensation management. 


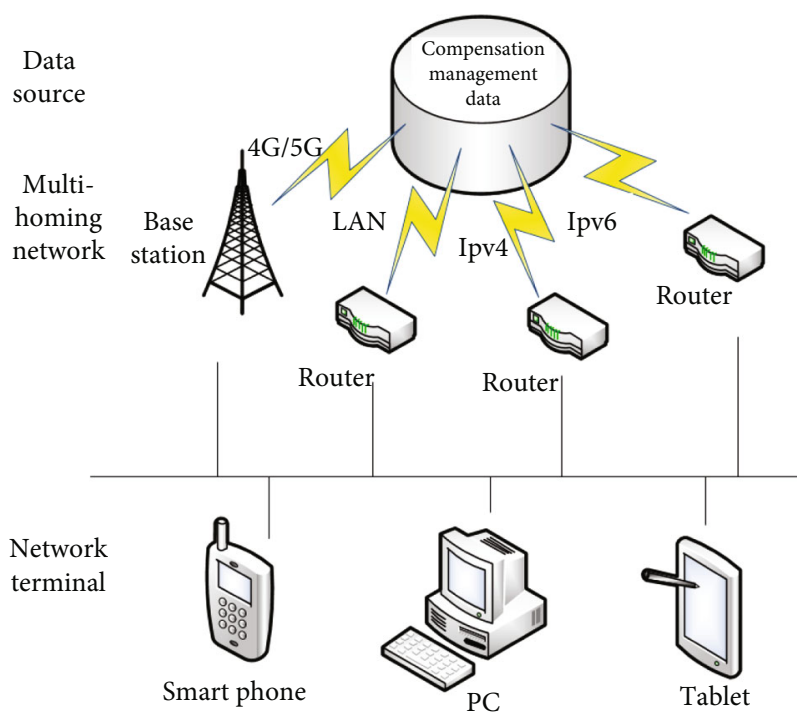

FIGURE 1: Multihoming network architecture of MNC-MIS.

3.2. Overall Structure Design of MNC-MIS. The overall structure of the system is divided into four layers, the interaction layer, application layer, data layer, and the external layer of other external systems, as shown in Figure 2.

3.2.1. External Layer. Due to the existence of the unified data platform of Donghua University, each MIS can exchange data through the unified data bus format. MIS for personal affairs, finance, and the retired are the systems with the most frequent data interaction with MNC-MIS. These MIS and other MIS of the university which may require compensation data support constitute the external layer.

MIS for personal affairs is mentioned in this layer. Can MNC-MIS directly use personnel information in MIS for personal affairs without having its own personal records database? The answer is no, because although the information in the two MIS is generally called personnel information, the content of the two is actually different. MIS for personal affairs focuses on all kinds of information related to personnel employment and management, while MNCMIS focuses on all kinds of information related to compensation. Of course, such public information as name, ID, professional title, and position must be reused by both MIS.

3.2.2. Interaction Layer. The interaction layer mainly includes the program modules that need to interact with users, and it is the entrance and exit of the system.

3.2.3. Application Layer. The application layer is the main part of the MIS and the core business logic of the whole system. All the internal processes of data management and processing occur in this layer. At the same time, this layer is the only one that has relations with all the other three layers.

3.2.4. Data Storage Layer. As the name suggests, the data storage layer is where the data is. The core data of this system is divided into two parts: one is the file personal records data and the compensation standard information associated with it. Which data is divided into two parts again, the data of working staff and the retired are stored in the two core databases of the data storage layer. The other core data is the actual payroll data, which also has a special core database. In addition, there are many kinds of subdatabases in this layer, such as historical information database, compensation reference database, and format of report form database. These sublibraries play the role of expanding the core library or supporting the storage of some business logic in the application layer.

3.3. System Function Module Design. On the basis of the overall structure design of the system, we give the system module design, as shown in Figure 3.

The function and significance of some important modules are as follows:

\section{(1) Various compensation promotion modules}

As mentioned in the first chapter, the compensation of public institutions has a strong policy focus, according to these policies, such as pay grade salary, Shanghai post allowance and other compensation items will be promoted naturally with the increase of teachers' working years. Of course, promotion cannot simply increase a fixed amount every year but has a series of constraint conditions. For example, promotion of pay grade salary needs to meet the constraints of passing the assessment, being on the job all year round, and not skipping a grade in the previous year, while the promotion of post allowance in Shanghai needs to meet the conditions of seniority being divided exactly by 5 , being on the job all year round, and seniority $\leq 35$.

All kinds of compensation promotion program modules in this MIS are written to solve the above problems. These modules have embedded judgment programs, which computerize the policy language, so that the computer can calculate the compensation after promotion according to the personal record database and compensation reference database. The working process of these program modules can also be seen in the data flow diagram in the following.

(2) Various time warning modules

In the work of compensation management in universities, due to the large number of teachers in universities, and the existence of dozens of policy which affects the change of compensation of faculty. For example, a new master's or doctor's degree student who has just joined the work will be transferred from an unassigned faculty member to a faculty of certain position level through position orientation after three months. Another example is that a teacher who has reached the age of 60 needs to retire, and his or her on-the-job pay will stop accordingly. There are still many such changes. Therefore, relying on human brain memory or form reminders is not timely or even omitted. The advantages of various time early warning modules in this MIS are reflected here. Through these modules, the system can accurately and timely give the administrator feedback reminder, so that the administrator can have enough time to deal with 


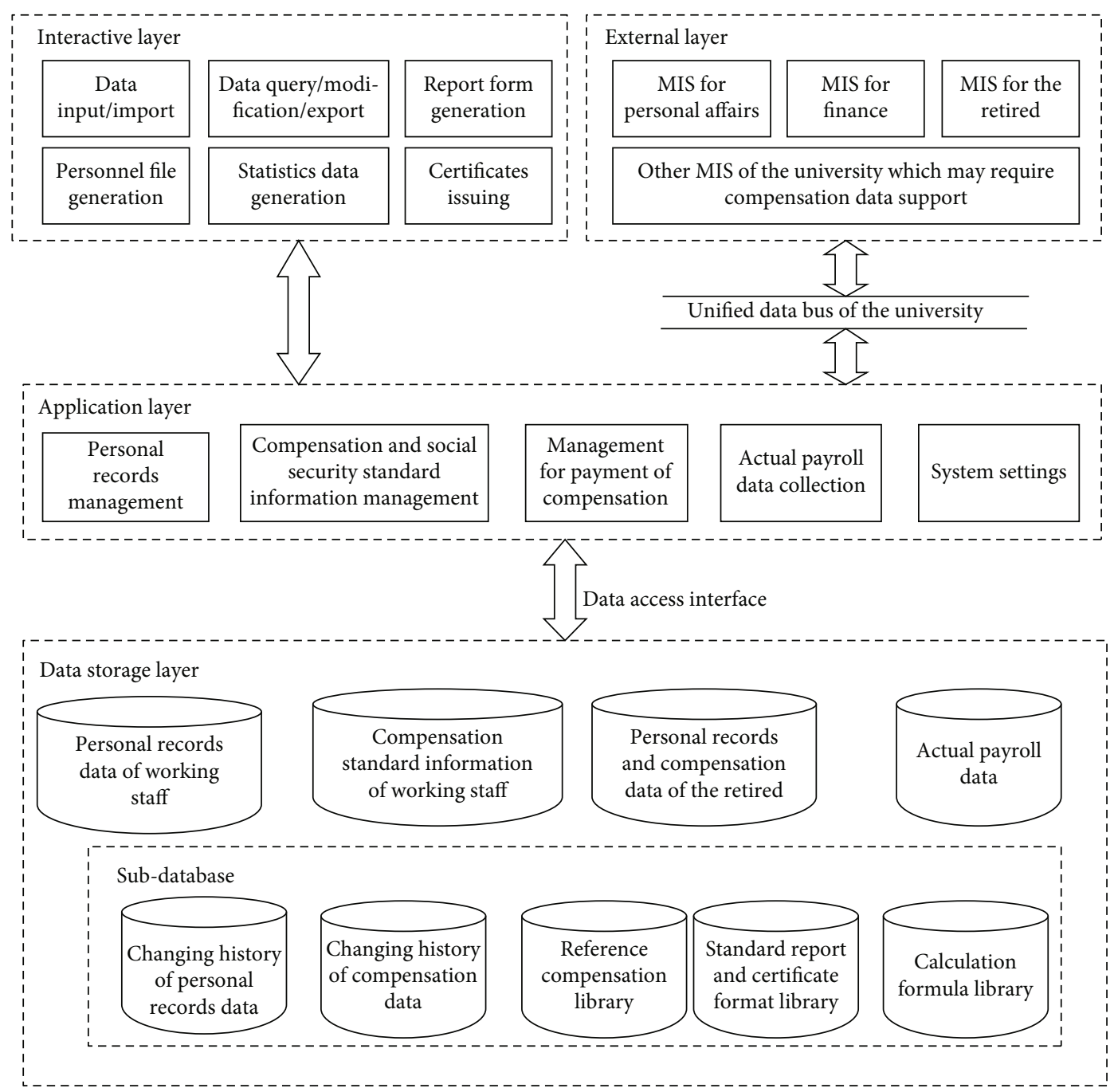

Figure 2: Overall structure of MNC-MIS.

the relevant changes, improve work efficiency, and also enhance the timeliness and accuracy of the work.

\section{(3) Personnel history data management}

The compensation policy of public institutions is not only numerous but also requires very meticulous. The whole experience of learning, work, and promotion of faculty is the basis and requirement of compensation promotion of public institutions. For example, in 2006, the starting grade of pay grade salary should be calculated according to the information of each teacher's number of years of schooling, number of years of working, and number of years of being in current position. Therefore, a good MIS for compensation must have the function of historical data record management.

The personnel history data management module shall be able to automatically record each employee's promotion time of title or position, and the compensation adjustment situation every time, and store them in a special database for long-term storage to provide various compensation adjustment policies. With the centralized management of the system, the compensation management has gone away from the original fragmented records, as well as a large number of complicated manual data checking process, and entered an efficient and automatic era.

(4) All kinds of statistics module and report generation module

Compensation in universities should be under the jurisdiction of higher authorities. Take Donghua University as an example, it is under the jurisdiction of Shanghai Municipal Government where it is located, and under the jurisdiction of the Ministry of Education of PRC. The above departments require our university to submit various compensation reports every quarter and every year. However, due to different management departments, these reports not only have different formats but also have different statistical calibers. In the absence of system support, we can only use a lot of time to split and summarize the original data of Excel in different forms according to different requirements, so as to meet the requirements of various reports.

Module for statistics of all kinds of indicators with module for report form generation of this MIS gives a perfect 


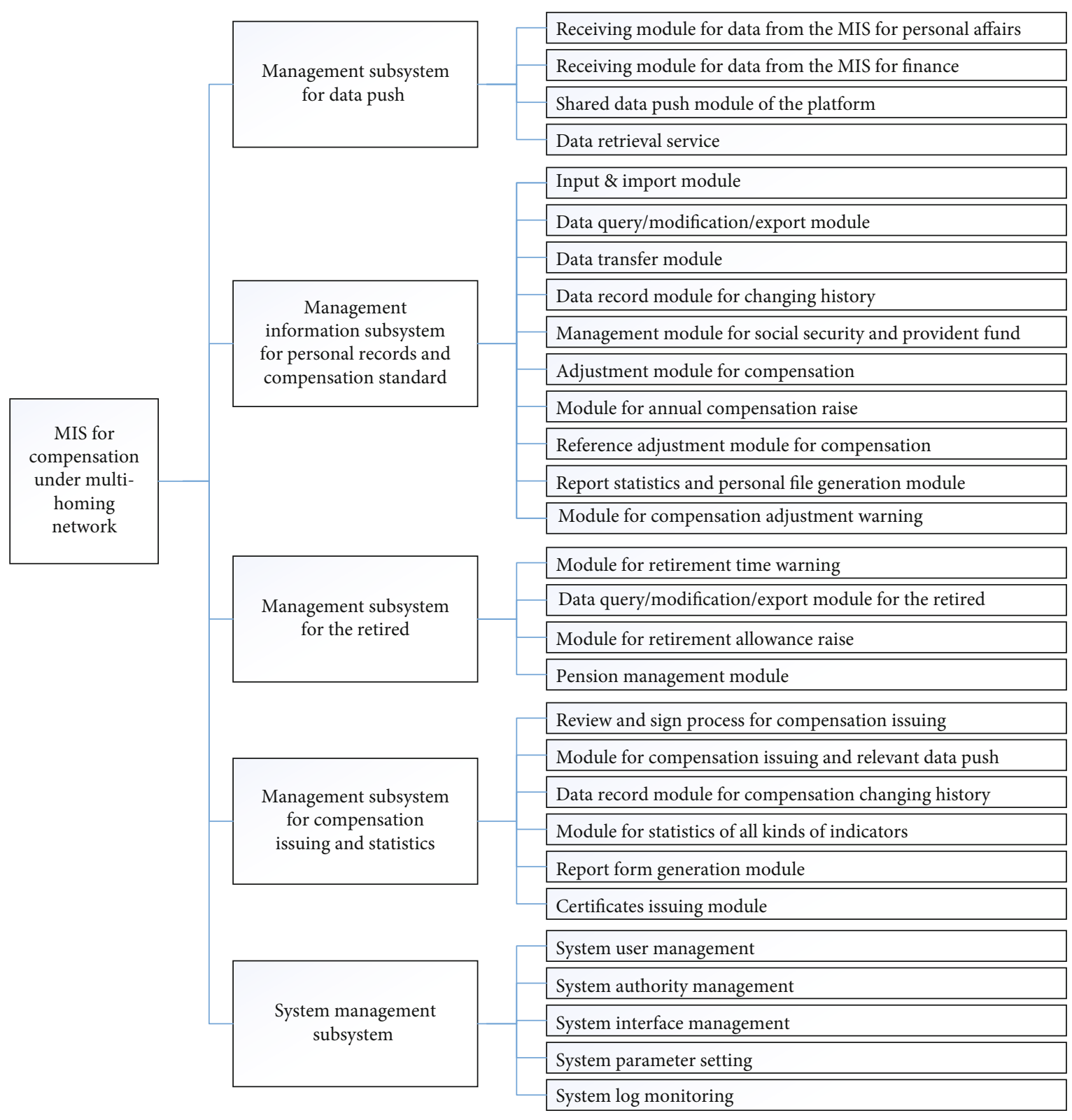

FIGURE 3: System function module design of MNC-MIS.

solution to this problem. These modules can use the data in both reference compensation database and actual payroll database and give different forms of reports according to the presupposed forms of report. At the same time, the system also has a format editor, which makes it convenient, accurate, and fast to report as you wish.

3.4. Design of System Data Flow. Data flow is the "blood" of each module of the system, so the design quality of data flow also reflects the quality of MNC-MIS and further reflects the level of compensation management in this university. The data flow diagram of MNC-MIS is shown in Figure 4.

The first thing to explain is that the program modules corresponding to entities 18 and 19 in the figure are connected with all the databases in the figure, but if they are lined with all the databases, the overall image will be very chaotic. Therefore, for the convenience of drawing, the "all database in this MIS" database flag represented by dotted line is drawn to refer to all databases in the diagram.

In this figure, we can see not only the operation condition of the data in this MIS but also the process of compensation management in Chinese universities. So, let us take the practical work of several processes in universities to help you understand and read the operation of the system data.

3.4.1. New Teachers Are Coming In. When a new teacher starts his or her career, he or she will first go through the check-in procedures in the personnel section of the human resource department and fill in his or her personal certificate information, study and work experience, and other information in the MIS for personal affairs. The MIS for personal affairs will push the above information related to 


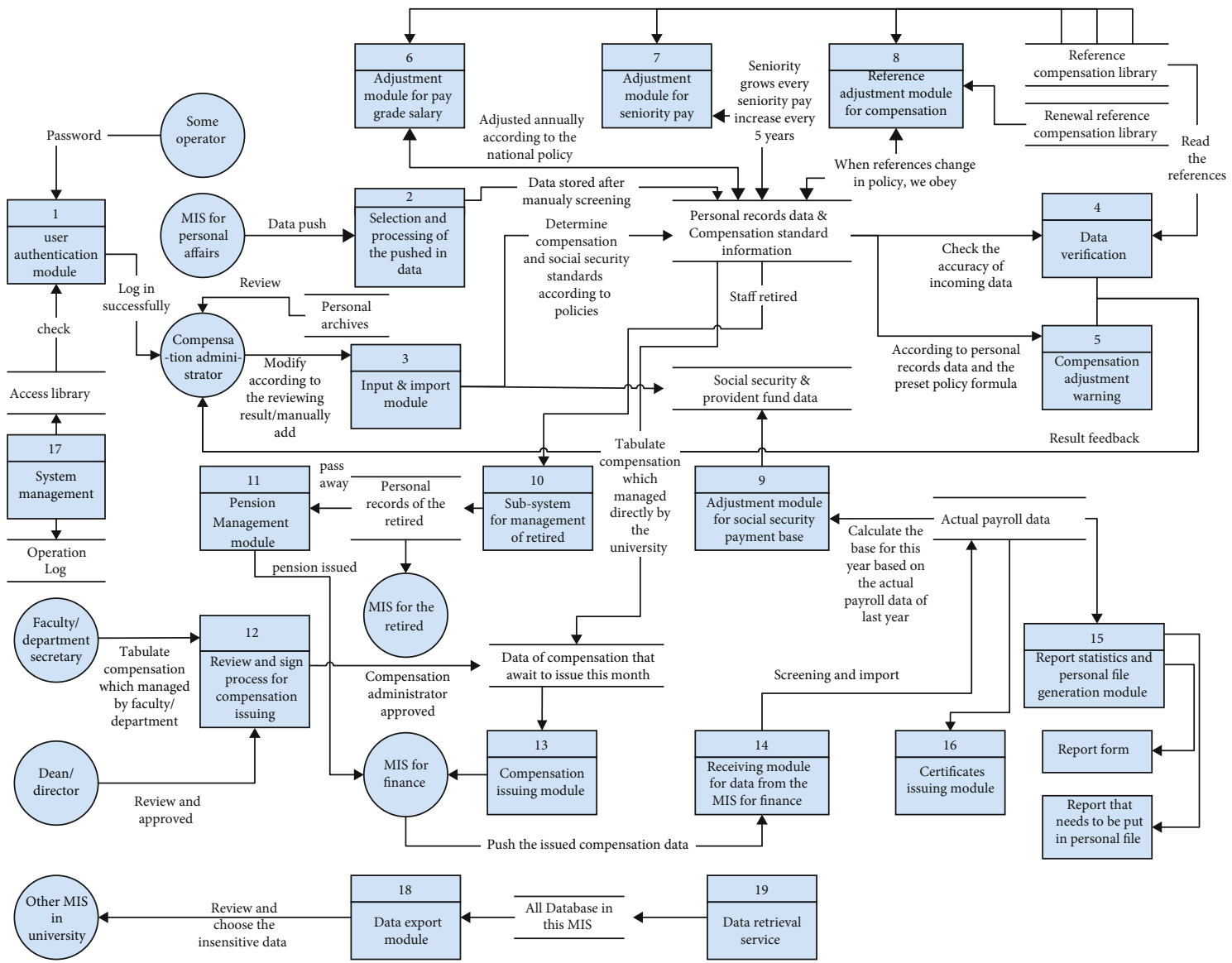

FIgURe 4: Data flow diagram of MNC-MIS.

determining the compensation standard, and through the selection and processing module shown in entity 2 , the selected information will be imported into the personal record database of the MIS. So, how to ensure the accuracy and authenticity of the information? This has to mention the personnel archive system with Chinese characteristics.

Personnel archives are paper materials recording personal identity, education background, qualifications, and other important contents. In China, each unit has a special file management organization. One cannot read its own personnel archive for life, and there are a series of systems to ensure the safety and confidentiality of archives in the process of storage and circulation. Therefore, if we want to determine the accuracy of personal information, we only need to view and refer to the personal file. The compensation administrator has the right to view personal archives.

In Figure 4, you can also see the process of compensation administrator consulting personal archives. According to the results of file reading, manually modify the received push data through entity 3 "input and import module." Then, the administrator should apply the compensation policy according to the above personal file information, manually calculate the standard value of each compensation, and fill in the system. After that, we can call the verification system of entity 4 to verify the compensation standard value. So why not use the computer to calculate the compensation standard in the first place? This is because some items in the compensation cannot be completely calculated by computer and must be judged by comprehensive manual work.

\subsubsection{Determination and Adjustment of Social Security} Payment Base. Through the above steps, a new employee's monthly compensation standard has been determined. According to this compensation standard, the social security payment base can be calculated. Enter the social security payment base into the social security management program of entity 9 , you can get the payment amount of social security and provident fund and store it. At the same time, the social security management program can automatically calculate the social security payment base of all employees in the current year according to the actual payroll data of the previous year and automatically complete the adjustment of the payment amount of the whole staff and the whole project once a year.

3.4.3. Monthly Compensation Issuing and Summarizing Process. There are two main functions of this MIS: one is the management of compensation standard, and the other is the management of monthly compensation issuing process. The system entity 12 module of review and sign process for compensation issuing is the entry of compensation payment process. According to the policy of Donghua University, the national compensation is managed by the compensation administrator of the university, while the school allowance is managed by the faculty/department 
and reviewed by the compensation administrator. This approval process is mainly for the school allowance. The faculty/department secretary makes a record of this part of the compensation and initiates the process. After the approval of the dean/director and the approval of the compensation administrator, this part of the compensation will enter into the payroll to be paid this month. In the figure, we can also see that the part of national compensation is directly recorded by the compensation administrator according to the information in the personal compensation standard database, combined with the actual situation of this month, and entered into the compensation database to be paid this month.

After obtaining all the compensation information to be paid this month, through the entity 13 compensation payment and docking module, the compensation information to be paid is pushed through the school unified platform, such as MIS for finance. After that, the compensation is paid to each teacher through the financial system.

Therefore, after the payment is completed, the paid out amount can be imported into the MIS through the closedloop system, and then, the actual payroll data can be compared with the data of compensation that await to issue. In addition, the actual payroll data is also an important basis for the program modules of entities 9, 15, and 16 .

3.4.4. Faculty Life Cycle Management. From the data flow process in Figure 4, we can see that from the beginning of employment to retirement or even death, the school will pay the teachers the relevant treatment in each period. Therefore, the whole life cycle management of employees is also a feature of personnel management in Chinese universities.

When the employee's compensation standard information system is transferred from the on-the-job management system, 10 employees will retire. Because our school has a special retiree management system, so this subsystem mainly plays the role of the link between this MIS and the retirement MIS and can complete some basic retiree information storage and management work. After that, the relevant information of retirees will be pushed to the special retirement MIS through the school platform for the management of the retirement management office.

When a retired teacher dies, the system will read the retiree's information through the entity 11 pension management module, calculate the teacher's pension amount according to the policy, and then transfer it to the financial MIS to complete the pension payment.

\section{The Function and Effect of MNC-MIS}

The system design fully shows the advantages of computer application in personnel management and provides the system users with as much convenience as possible, such as fast retrieval, comprehensive data, closely following policy, closed-loop management, and personalized operation.

(1) The data format is unified, and the data exchange is accurate and standard

The data format is unified in two aspects:
First, on the aspect of whole school. The system follows the data specification of the unified information platform of the school and can achieve the data docking with other MIS of the school through the unified data bus of the university information platform. The system level data exchange is fast and accurate.

Second, on the aspect of the system itself. There are several main databases and several subdatabases in the system. All databases follow the data protocol agreed in the system. Through the design of this specification, not only the unity of the system data is ensured but also the scalability of the system data is ensured.

(2) The design of data structure emphasizes integrity and comprehensiveness, which meets the needs of compensation management in universities

Data structure design in line with the principle of "can be complete, can be detailed," let the data field be as complete as possible. Data management is based on the principle of more and more, recording every change and every adjustment truthfully and carefully. Because every field, every adjustment, may have an impact on a teacher's subsequent compensation. For example, in some specific cases, two teachers who are consistent in all aspects have only one promotion time difference of one month. This month may bring a gap between the two teachers' salaries in a future compensation adjustment. The emphasis of data integrity and comprehensiveness in this system is the best preparation for such a situation.

(3) To achieve the machine language of compensation policy at all levels and fully achieve the policy adjustment of university compensation

This paper has repeatedly emphasized the characteristics of a strong policy focus of university compensation. Governments at all levels, such as the state, provinces, and cities, have issued a lot of compensation policies, which have been edited into a book and distributed to the compensation administrators of colleges and universities. This system transforms these policies written in natural language into machine language, which means that the computer has learned these policies. Then, the computer can complete all kinds of compensation promotion and adjustment according to these policies.

At the same time, the compensation policy is constantly changing, and the system also retains the interface, allowing the entry of new machine language policy procedures without changing the source code, ensuring the continuity and scalability of the policy.

(4) The closed-loop management of the whole process of compensation management is achieved

The whole process of university compensation management can be closed-loop in this system. From the compensation starting of new employees, to the determination of compensation standard, to the payment of compensation, to the payment of social security and provident fund, to 
the summary of actual payroll data, to the review of compensation standard, and finally to statistics and statements. The data of compensation achieves a big close loop. In these large cycles, there are also some small cycles, such as the compensation standard verification process and the social security payment base determination process, which are all small closed-loop cycles in the system. The process of data circulation is the process of compensation management, and the comprehensive informatization of these processes also marks the comprehensive informatization of compensation management, which is the embodiment of efficient, accurate, and professional compensation management in colleges and universities.

(5) Data statistics and report results are instant, convenient, and accurate

Thanks to the unified data format, comprehensive data content, and sufficient policy learning, the process of data statistics and data report generation is efficient and accurate. This process is like a sports car with a well-maintained engine, full oil tank, and shiny body parked in the garage and ready to go. When the driver wants to drive, he or she can go straight away and become a beautiful scenery on the road.

(6) Definable and extensible operation interface, flexible personality

The menu module of MIS supports creation and adjustment, and the operation interface also supports multidimensional configuration. Through the personalized design and adjustment, we can create the most user-friendly interface and give users a good experience.

\section{(7) The factors affecting MNC-MIS}

Although MNC-MIS has many advantages, some factors will affect the performance of the system. For example, the bandwidth and security [21] of each network, the performance of the server, the completeness and accuracy of the original salary data, the clarity of salary-related policies, and the speed of policy communication.

To sum up, the MNC-MIS is a system engineering with high design requirements, great development difficulty, and strong technical requirements. This MIS is designed based on my five years' experience in the front-line of compensation management work. The purpose of this MIS is to fully solve the practical problems of compensation management in universities, truly help the work of compensation administrators, unify the fragmented compensation management works, and comprehensively improve the level of compensation management. It has a strong popularization and signification for reference for the compensation management work of similar universities.

\section{Conclusions and Future Work}

This paper designs a kind of management information system for compensation under multihoming network architec- ture. Various compensation promotion module, time early warning module, personnel history data management module, statistics module, and report generation module and the detailed data flow have been designed. This MIS can achieve the functions of user authority management, compensationrelated information query, information statistics, and system maintenance and solve the problems of not comprehensive function, poor report processing function ability, cumbersome query, and statistics of the existing compensation management system. Using this MIS can achieve efficient intelligent management of compensation data.

In future work, we will strive to improve the big data processing technology of the system, improve the data analysis and processing capacity of the system, improve network performance with advanced methods [22], and adopt or develop advanced artificial intelligence algorithms to further improve the intelligent decision-making and suggestion push function of the system. Moreover, we will seek to exchange information with other universities to establish a general compensation management platform and improve the overall compensation data management ability.

\section{Data Availability}

The data used to support the findings of this study are available from the corresponding author upon reasonable request.

\section{Conflicts of Interest}

The authors declare that they have no conflicts of interest.

\section{Acknowledgments}

This work was supported in part by the National Natural Science Foundation of China (Grant No. 61903078) and 2020 Donghua University Higher Education Planning Special Topic Project (Research on Performance Wage Distribution Method Based on the Sense of Pay Fairness, No. 20699-0243030).

\section{References}

[1] J. Chen, Z. Lv, and H. Song, "Design of personnel big data management system based on blockchain," Future Generation Computer Systems, vol. 101, pp. 1122-1129, 2019.

[2] G. Donati and C. Woolston, "Information management: data domination," Nature, vol. 548, no. 7669, pp. 613-614, 2017.

[3] J. Hou, Z. Wang, X. Liu et al., "Public health education at China's higher education institutions: a time-series analysis from 1998 to 2012," Bmc Public Health, vol. 18, no. 1, p. 679, 2018.

[4] L. Xu, A. Nallanathan, J. Yang, and W. Liao, "Power and bandwidth allocation for cognitive heterogeneous multi-homing networks," IEEE Transactions on Communications, vol. 66, no. 1, pp. 394-403, 2018.

[5] K. Poularakis, G. Iosifidis, and L. Tassiulas, "Joint deployment and pricing of next-generation wifi networks," IEEE Transactions on Communications, vol. 67, no. 9, pp. 6193-6205, 2019. 
[6] V. Kumar and N. B. Mehta, "Modeling and analysis of differential cqi feedback in $4 \mathrm{~g} / 5 \mathrm{~g}$ ofdm cellular systems," IEEE Transactions on Wireless Communications, vol. 18, no. 4, pp. 2361-2373, 2019.

[7] A. D'Alconzo, I. Drago, A. Morichetta, M. Mellia, and P. Casas, "A survey on big data for network traffic monitoring and analysis," IEEE Transactions on Network and Service Management, vol. 16, no. 3, pp. 800-813, 2019.

[8] S. Babu, A. Rajeev, and B. S. Manoj, "A medium-term disruption tolerant SDN for wireless TCP/IP networks," IEEE Transactions on Network and Service Management, vol. 17, no. 4, pp. 2318-2334, 2020.

[9] L. K. Indumathi and D. S. Punithavathani, "Performance improvement of proxy mobile ipv6 for the support of multihoming," Wireless Personal Communications: An International Journal, vol. 96, no. 2, pp. 1653-1672, 2017.

[10] I. Cho, K. Okamura, T. W. Kim, and C. S. Hong, "Performance analysis of IP mobility with multiple care-of addresses in heterogeneous wireless networks," Wireless Networks, vol. 19, no. 6, pp. 1375-1386, 2013.

[11] T. Dreibholz, E. P. Rathgeb, I. Rüngeler, R. Seggelmann, M. Tüxen, and R. R. Stewart, "Stream control transmission protocol: past, current, and future standardization activities," IEEE Communications Magazine, vol. 49, no. 4, pp. 82-88, 2011.

[12] A. Keranen, "This document specifies two transport modes for host identity protocol (HIP) signaling messages that allow conveying them over encrypted connections initiated with the host identity protocol," Radiology, vol. 98, no. 98, pp. 605-610, 2015.

[13] Y. Qiao, E. Fallon, J. Murphy, L. Murphy, Z. Shi, and A. Hanley, "Transmission scheduling for multi-homed transport protocols with network failure tolerance," Telecommunication Systems, vol. 43, no. 1-2, pp. 39-48, 2010.

[14] J. Cao, Z. Li, Q. Luo, and Q. Hao, "Research on the construction of smart university campus based on big data and cloud computing," in 2018 International Conference on Engineering Simulation and Intelligent Control (ESAIC), pp. 351-353, Hunan, China, 2018.

[15] K. Melendez, A. Dávila, and M. Pessoa, "Information technology service management models applied to medium and small organizations: a systematic literature review," Computer Standards \& Interfaces, vol. 47, pp. 120-127, 2016.

[16] L. Zhong, "Monitoring function design of radio monitoring management system based on C/S architecture," in 2019 10th International Conference on Information Technology in Medicine and Education (ITME), pp. 424-428, Qingdao, China, 2019.

[17] M. Agiwal, H. Kwon, S. Park, and H. Jin, “A survey on 4G-5G dual connectivity: road to $5 \mathrm{G}$ implementation," IEEE Access, vol. 9, pp. 16193-16210, 2021.

[18] W. Kim, J. Park, J. Jo, and H. Lim, “Covert jamming using fake ACK frame injection on IEEE 802.11 wireless LANs," IEEE Wireless Communications Letters, vol. 8, no. 5, pp. 15021505, 2019.

[19] J. Xie and U. Narayanan, "Performance analysis of mobility support in IPv4/IPv6 mixed wireless networks," IEEE Transactions on Vehicular Technology, vol. 59, no. 2, pp. 962-973, 2010.

[20] J. Q. Zhu, Y. L. Ban, Y. Zhang et al., “A novel capacitive coupler array with free-positioning feature for mobile tablet applica- tions," IEEE Transactions on Power Electronics, vol. 34, no. 7, pp. 6014-6019, 2019.

[21] H. N. Noura, R. Melki, M. Malli, and A. Chehab, "Lightweight and secure cipher scheme for multi-homed systems," Wireless Networks, 2020.

[22] A. AI-Najjar, F. H. Khan, and M. Portmann, "Network traffic control for multi-homed end-hosts via SDN," IET Communications, vol. 14, no. 19, pp. 3312-3323, 2020. 